\title{
Hydroxyl Influence on Adsorption and Lubrication of Ultra-Thin Aqueous Triblock Copolymer Lubricant
}

\author{
Thi D. Ta*, A. Kiet Tieu, Bach H. Tran
}

School of Mechanical, Materials Mechatronic and Biomedical Engineering, Faculty of Engineering and Information Sciences (EIS), University of Wollongong, Northfield Avenue, Wollongong, NSW, 2522, Australia 


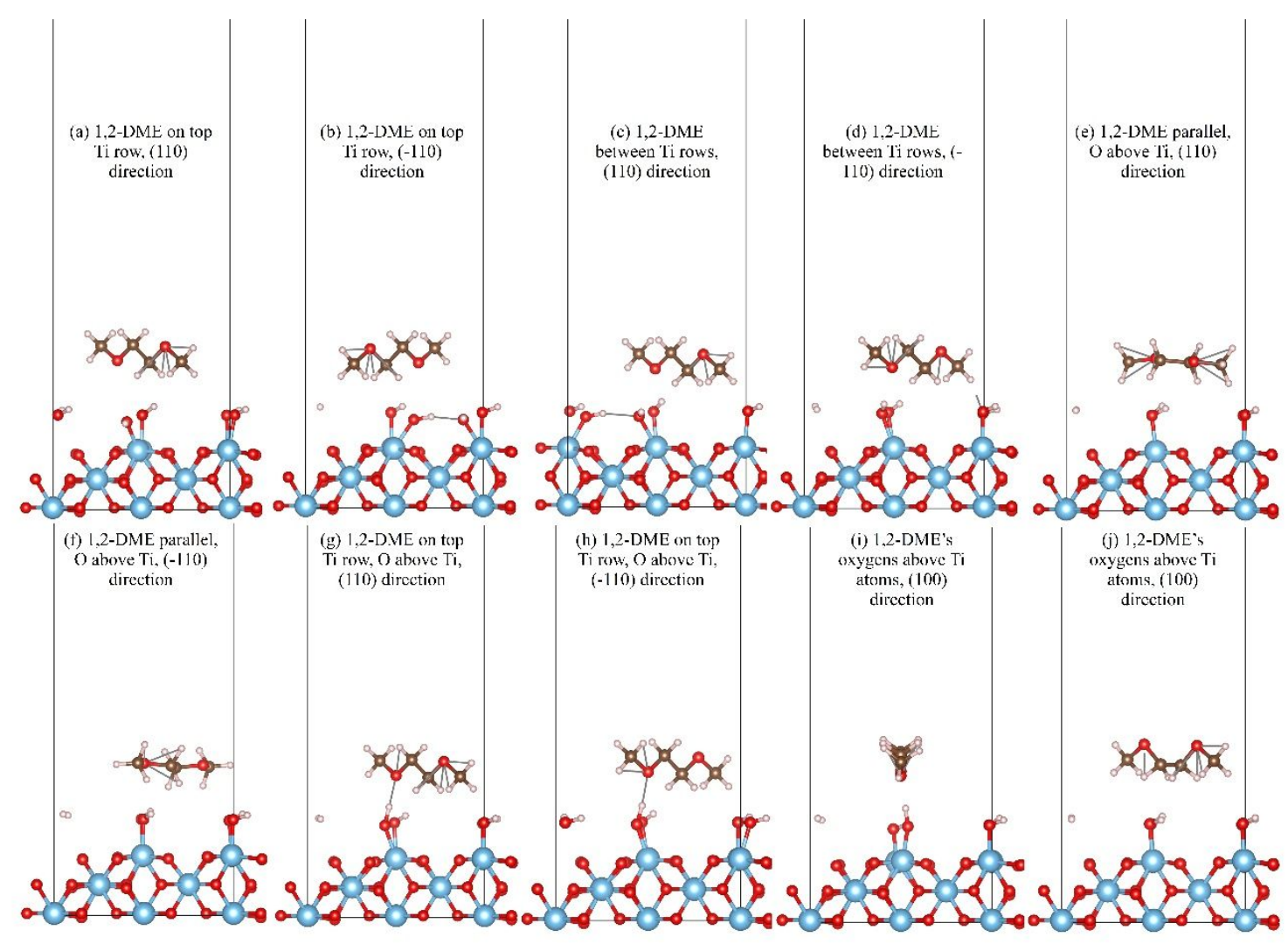

Fig. S1 Different adsorption configurations of 1,2-DME onto hydrated $\mathrm{TiO}_{2}(001$ surface. Marine, red, grey, and white colors present $\mathrm{Ti}, \mathrm{O}, \mathrm{C}$, and $\mathrm{H}$, respectively. 


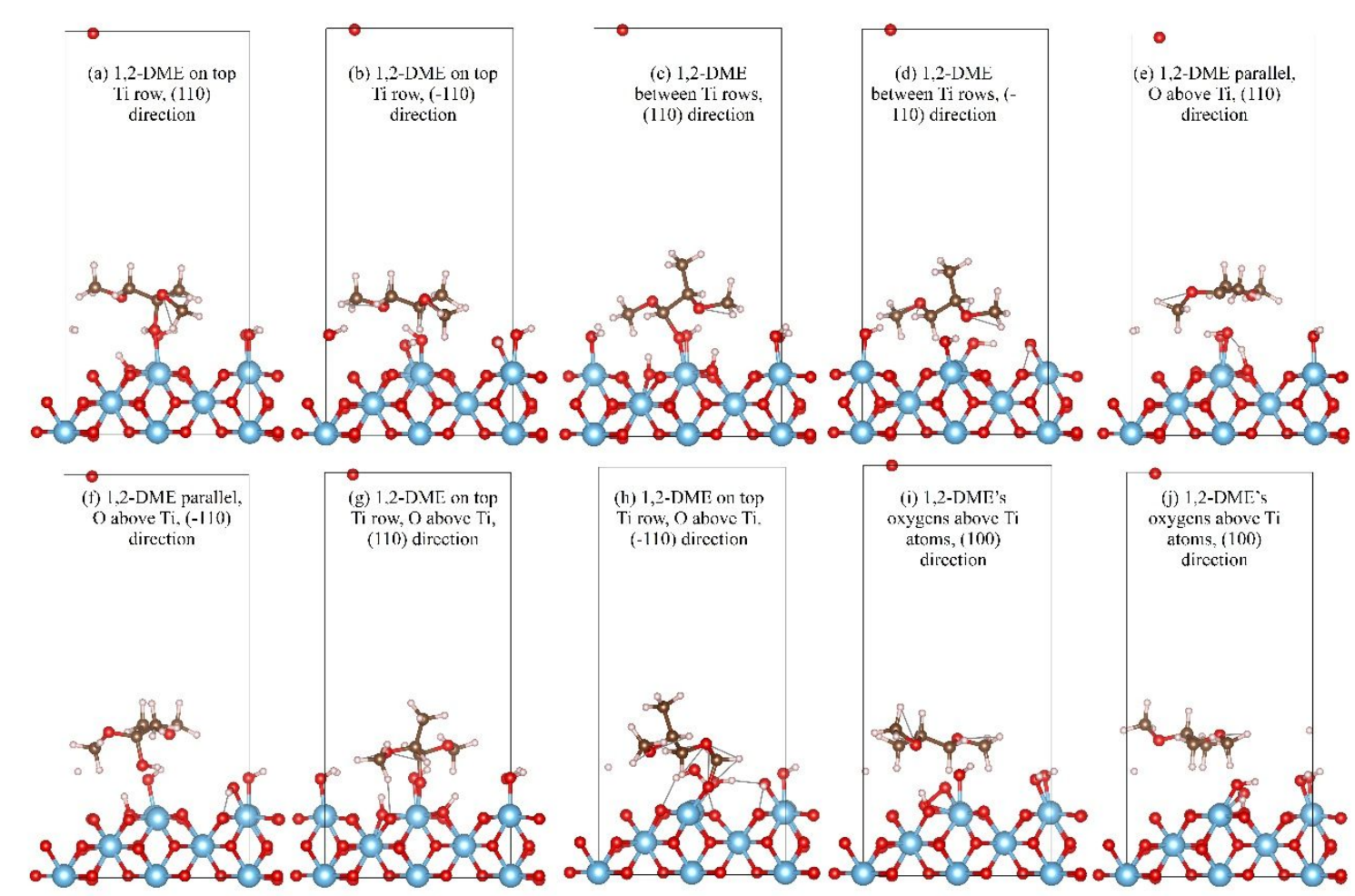

Fig. S2 Different adsorption configurations of 1,2-DMP onto hydrated $\mathrm{TiO}_{2}(001)$ surface. Marine, red, grey, and white colors present $\mathrm{Ti}, \mathrm{O}, \mathrm{C}$, and $\mathrm{H}$, respectively.

\begin{tabular}{|c|c|c|c|c|c|c|}
\hline \multirow{2}{*}{$\frac{\text { Surface }}{\mathrm{TiO}_{2}(001)}$} & \multicolumn{2}{|c|}{ Lattice sizeLubricant } & \multicolumn{2}{|c|}{ Surface domain No. surface atoms } & \multicolumn{2}{|c|}{ No. lubricant atomsTotal No. atoms } \\
\hline & $\mathrm{p}(2 \times 2 \times 1)$ & 1,2-DME & $9.19 \times 9.19 \AA^{2}$ & $12 \mathrm{Ti}+24 \mathrm{O}$ & 16 & 52 \\
\hline $\mathrm{TiO}_{2}(001)$ & $\mathrm{p}(2 \times 2 \times 1)$ & 1,2-DMP & $9.19 \times 9.19 \AA^{2}$ & $12 \mathrm{Ti}+24 \mathrm{O}$ & 19 & 55 \\
\hline $\mathrm{TiO}_{2}(001)-\mathrm{OH}$ & $\mathrm{p}(2 \times 2 \times 1)$ & 1,2-DME & $9.19 \times 9.19 \AA^{2}$ & $12 \mathrm{Ti}+24 \mathrm{O}+4 \mathrm{O}(\mathrm{OH})+12 \mathrm{H}(\mathrm{OH})$ & 16 & 68 \\
\hline $\mathrm{TiO}_{2}(001)-\mathrm{OH}$ & $\mathrm{p}(2 \times 2 \times 1)$ & 1,2-DMP & $9.19 \times 9.19 \AA^{2}$ & $12 \mathrm{Ti}+24 \mathrm{O}+4 \mathrm{O}(\mathrm{OH})+12 \mathrm{H}(\mathrm{OH})$ & 19 & 71 \\
\hline $\mathrm{TiO}_{2}(001)$ & $\mathrm{p}(2 \times 2 \times 1)$ & $15 \mathrm{H}_{2} \mathrm{O}$ & $9.19 \times 9.19 \AA^{2}$ & $12 \mathrm{Ti}+24 \mathrm{O}$ & 45 & 97 \\
\hline $\mathrm{TiO}_{2}(001)$ & $\mathrm{p}(2 \times 2 \times 1)$ & $1,2-\mathrm{DME}+15 \mathrm{H}_{2} \mathrm{O}$ & $9.19 \times 9.19 \AA^{2}$ & $12 \mathrm{Ti}+24 \mathrm{O}$ & 61 & 113 \\
\hline $\mathrm{TiO}_{2}(001)$ & $\mathrm{p}(2 \times 2 \times 1)$ & $1,2-\mathrm{DMP}+15 \mathrm{H}_{2} \mathrm{O}$ & $9.19 \times 9.19 \AA^{2}$ & $12 \mathrm{Ti}+24 \mathrm{O}$ & 64 & 100 \\
\hline $\mathrm{TiO}_{2}(110)$ & $\mathrm{p}(2 \times 4 \times 2)$ & $42 \mathrm{H}_{2} \mathrm{O}$ & $12.99 \times 14.80 \AA^{2}$ & $40 \mathrm{Ti}+80 \mathrm{O}$ & 126 & 246 \\
\hline $\mathrm{TiO}_{2}(110)$ & $\mathrm{p}(2 \times 4 \times 2)$ & $1,2-\mathrm{DME}+42 \mathrm{H}_{2} \mathrm{O}$ & $012.99 \times 14.80 \AA^{2}$ & $40 \mathrm{Ti}+80 \mathrm{O}$ & 142 & 262 \\
\hline $\mathrm{TiO}_{2}(110)$ & $\mathrm{p}(2 \times 4 \times 2)$ & $1,2-\mathrm{DMP}+42 \mathrm{H}_{2} \mathrm{O}$ & $12.99 \times 14.80 \AA^{2}$ & $40 \mathrm{Ti}+80 \mathrm{O}$ & 145 & 265 \\
\hline
\end{tabular}



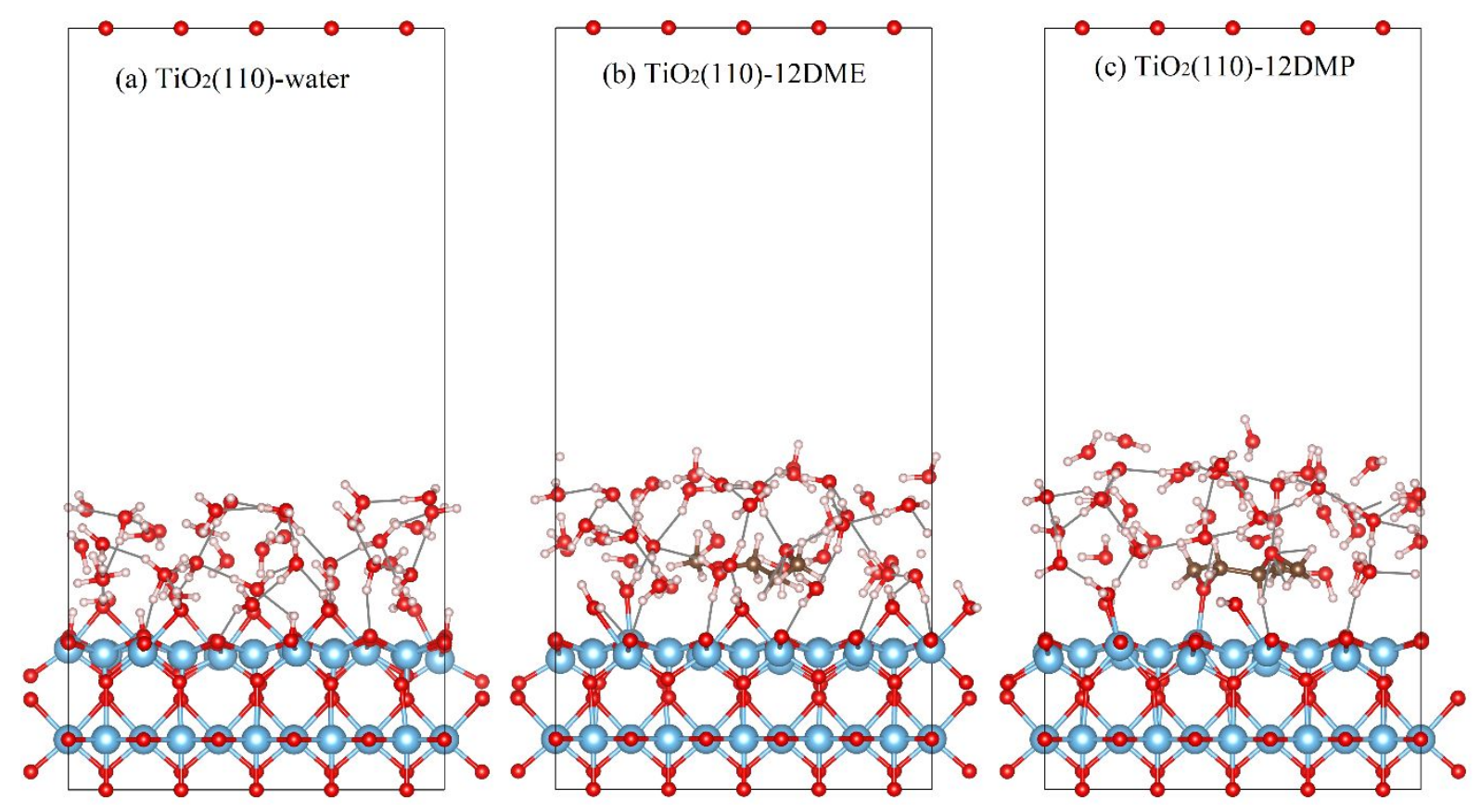

Fig. S3 Optimized molecular structure of (a) water; (b) 1,2-DME in water, and (c) 1,2-DMP in water onto $\mathrm{TiO}_{2}(110)$ surface. Marine, red, grey, and white colors present Ti, O, C, and $\mathrm{H}$, respectively. 
Table S2

Structure, mass ratio of PEO block, weight percentage of Pluronics in water solution, number of molecules and $\mathrm{H}_{2} \mathrm{O}$ for different types of solution.

\begin{tabular}{ccrccc}
\hline Pluronic & Structure & $\begin{array}{c}\text { PEO } \\
\text { \%wt }\end{array}$ & Pluronic wt\% & no. molecules & no. water \\
\hline 17R2 & $\mathrm{PPO}_{15}-\mathrm{PEO}_{10}-\mathrm{PPO}_{15}$ & 20 & 16 & 76 & 47446 \\
L62 & $\mathrm{PEO}_{6}-\mathrm{PPO}_{32}-\mathrm{PEO}_{6}$ & 20 & 16.2 & 68 & 47440 \\
\hline
\end{tabular}
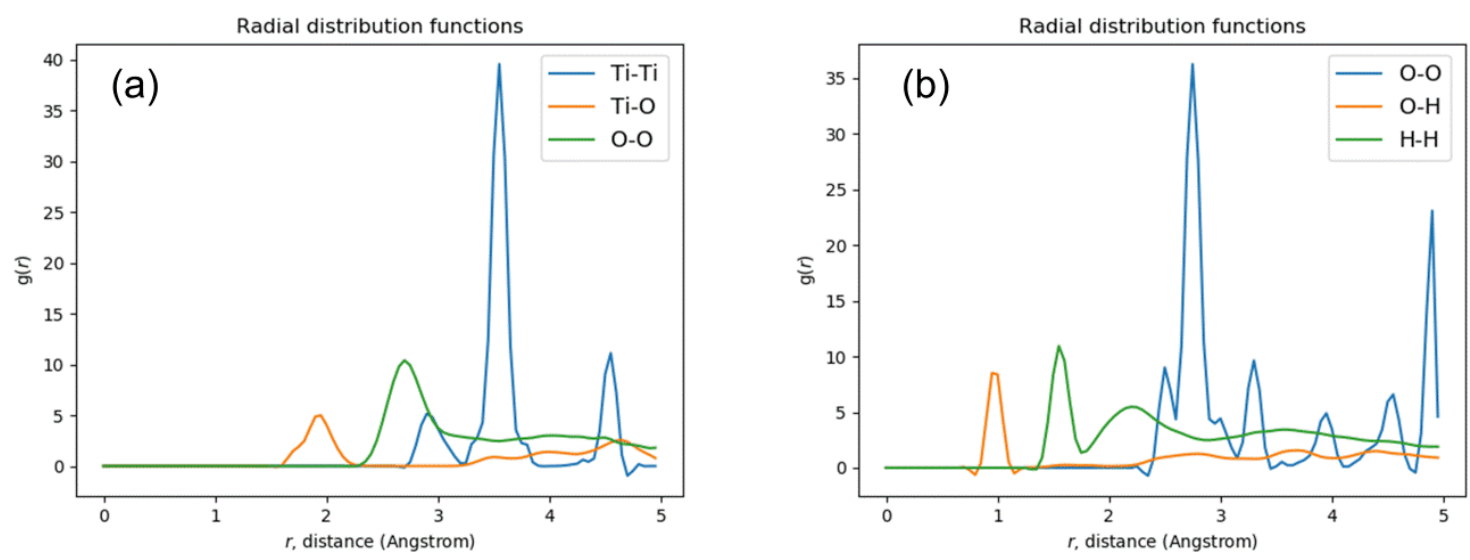

Fig. S4 Radial distribution functions g(r) of: (a) Ti- $\mathrm{O}_{w}$; and (b) $\mathrm{O}_{\text {sur }}-\mathrm{H}_{\mathrm{w}}$ pairs for DME-aqueous solution. 